\title{
Emisario submarino de Mar del Plata (Argentina): ¿Cómo impactó su construcción en la comunidad bentónica intermareal?
}

\author{
Graciela V. Cuello; Elizabeth N. Llanos; Griselda V. Garaffo² \& María L. \\ $\mathrm{JAUBET}^{2, \mathbb{}}$ \\ ${ }^{1}$ Grupo Bioindicadores Biológicos, Departamento de Ciencias Marinas, Facultad de Ciencias Exactas y Naturales, Universidad \\ Nacional de Mar del Plata, Mar del Plata, Argentina. ${ }^{2}$ Instituto de Investigaciones Marinas y Costeras, Universidad Nacional \\ de Mar del Plata (IIMyC-UNMdP)-Consejo Nacional de Investigaciones Científicas y Técnicas (CONICET).
}

\begin{abstract}
Resumen. Una solución adecuada al problema que deben resolver muchos países con respecto al tratamiento y el vertido de aguas cloacales, y su potencial impacto sobre el ambiente, podría ser verterlas por medio de emisarios submarinos. Hasta diciembre de 2014, los residuos cloacales de Mar del Plata se vertían de forma directa a la zona intermareal, sin tratamiento previo. En el año 2009 se comenzó a construir el emisario submarino (ES); la obra se inició con la incorporación de dos escolleras de abrigo y un frente de atraque. El ES, inaugurado en diciembre del 2014, descarga las aguas residuales sobre el submareal (4 km mar adentro). El objetivo de este trabajo fue evaluar los cambios en la estructura de la comunidad bentónica intermareal durante las distintas fases del proceso de construcción del ES (Antes, Durante y Después). Se consideraron tres sitios de muestreo localizados a diferentes distancias del efluente cloacal. Los resultados mostraron cambios en la comunidad bentónica intermareal como respuesta a la puesta en funcionamiento del ES. La abundancia de las especies sensibles a la contaminación aumentó en la etapa posterior a la puesta en funcionamiento del ES. Si bien la riqueza de especies alcanzó su pico máximo durante la fase de construcción del ES, la equitatividad y la diversidad aumentaron en la etapa posterior. Se observaron agrupamientos según la similitud en la composición específica según el factor etapa (Antes). Sin embargo, no se observaron agrupamientos con respecto a los sitios. En conclusión, los resultados obtenidos mostraron los cambios ocurridos en la estructura comunitaria intermareal asociados al nuevo vertido de aguas residuales generado por el emisario submarino construido en la ciudad.
\end{abstract}

[Palabras clave: contaminación orgánica, desagües cloacales, efluente submareal]

\begin{abstract}
AвsтRACt. Submarine outfall of Mar del Plata (Argentina): how did the construction impact on the intertidal benthic community? An adequate solution to the problem many countries must solve with respect to the treatment and discharge of sewage water, and its potential impacts on the environment, could be discharging them by means of submarine outfalls. Until December 2014, wastewater from Mar del Plata city was discharged untreated directly to the intertidal zone. In 2009, the construction of the submarine outfall (SO) began; two breakwaters and a docking front were built. The SO was inaugurated in December 2014 and the wastewater discharges directly over the subtidal (4 km offshore). The aim of this study was to evaluate the changes in the structure of the intertidal benthic community during the different phases of the SO construction [Before (A), During $(\mathrm{Du})$ and After (De)]. Three sampling sites were located at different distances from the sewage effluent. The results showed changes in the intertidal benthic community in response to the functioning of the SO. The abundance of species sensitive to pollution increased in the posterior phase of the SO construction. Although the species richness peaked during the construction phase of the ES, evenness and diversity index increased in the subsequent phase (After). Groupings were observed according to the similarity in the specific composition according to the phase factor (Before, During and After). However, grouping of species according to the sites were not observed. In conclusion, the results obtained showed the changes occurred in the intertidal community structure associated with the new wastewater discharge generated by the submarine outfall built in the city.
\end{abstract}

[Keywords: organic pollution, sewage impact, subtidal effluent]

Editor asociado: Pedro Daleo
Recibido: 5 de Junio de 2018

Aceptado: 25 de Octubre de 2018 


\section{INTRODUCCIÓN}

Una solución adecuada al problema que deben resolver muchos países con respecto al tratamiento y el vertido de aguas cloacales, y su potencial impacto sobre el ambiente, podría ser verterlas por medio de emisarios submarinos. Estos medios de descarga proveen una tecnología eficiente, segura y relativamente económica para la disposición final de aguas residuales, y pueden alcanzar los objetivos de calidad (i.e., bajos niveles de microorganismos patógenos, baja turbidez, bajos valores de nutrientes disueltos, entre los más importantes), además de minimizar los impactos adversos sobre el ambiente, la ecología y a la salud pública (Salas 2000).

Se estima que menos de $6 \%$ de la población total de América Latina y el Caribe descargan sus vertidos por medio de emisarios submarinos (Salas 2000) y tienen tratamiento de las aguas residuales (Madera et al. 2005). Puerto Rico es uno de los países con mayor inversión en la disposición final de aguas servidas, con un total de 15 emisarios submarinos construidos. Allí se aplican los criterios más modernos de diseño y se llevan a cabo estudios post-operativos de calidad de agua para asegurar el desempeño y cumplimiento de tales criterios. En Brasil, a pesar de que existen emisarios submarinos construidos, generalmente no se aplica tratamiento a las aguas residuales (Salas 2000). Chile también cuenta con el funcionamiento de emisarios con tecnologías disponibles para un saneamiento primario y secundario de los efluentes, pero existe una carencia de tratamiento avanzado y de procesos de desinfección (Barañao and Tapia 2004).

En la Argentina, actualmente se conocen dos localidades que cuentan con el sistema de desagües por emisarios submarinos: 1) Berazategui $\left(34^{\circ} 46^{\prime} 04^{\prime \prime} \mathrm{S}-58^{\circ} 12^{\prime} 48^{\prime \prime} \mathrm{O}\right.$ ) (con cañerías de $2.5 \mathrm{~km}$ de largo colocadas sobre una zanja dragada en el lecho del Río de la Plata), ubicado en la cuenca Berazategui (Buenos aires), que drena los efluentes cloacales de aproximadamente trece municipios hacia la planta de tratamiento Wilde y luego los arroja en el Río de la Plata a través de dicho emisario (Montera 2012); y 2) Mar del Plata (con cañerías de $4 \mathrm{~km}$ de largo colocada sobre el lecho marino) (www.osmgp.gov.ar).

Hasta diciembre de 2014, los residuos cloacales de la ciudad de Mar del Plata se vertían directamente al mar (zona intermareal), con sólo un pretratamiento (Vallarino et al. 2002; Elías et al. 2003, 2006, 2009, 2015; Vallarino and Elías 2006; Vallarino 2013; Sánchez 2014). La descarga de las aguas se realizaba por medio de un efluente doméstico ubicado a $9 \mathrm{~km}$ al norte del centro de la ciudad, de forma directa sobre el sector intermareal de sustrato rocoso (plataforma de abrasión de loess consolidado). Dicha área se caracterizaba por la presencia de densos bancos del mitílido Brachidontes rodriguezii y la comunidad asociada (Vallarino 2013). Dentro de esta última se encontraban los moluscos Mytilus edulis (=M. platensis), Shiponaria lessoni y los crustáceos Balanus glandula, Idotea balthica, Sphaeroma serratum, Monocorophium insidiosum (Vallarino et al. 2002; Vallarino 2013). En cuanto a los poliquetos, su distribución presentaba el comportamiento clásico en relación a un gradiente orgánico: especies tolerantes distribuidas mayormente en las áreas muy disturbadas (i.e., más cercanas a la descarga cloacal) y especies sensibles distribuidas en áreas no impactadas (Pocklington and Wells 1992). Entre las especies tolerantes se encontraban especies como Capitella "capitata" sp., Capitella sp., Boccardia spp., Cirratulus cirratus, Syllis prolixa, entre otros (Vallarino 2013).

En el año 2009 se comenzó a construir el emisario submarino (ES), que consiste en un efluente de $2 \mathrm{~m}$ de diámetro y $4 \mathrm{~km}$ de longitud, más una sección de difusores de 540 $\mathrm{m}$ con 130 boquillas (o difusores) que descarga las aguas residuales directamente sobre el área submareal. El período de construcción del ES coincidió con un acontecimiento único a nivel mundial y sin precedentes en la zona de estudio: la presencia de un poliqueto invasor Boccardia proboscidea (oriundo de California) (Hartman 1941). Su invasión fue detectada por primera vez en primavera de 2008 por medio del desarrollo de los arrecifes: enormes estructuras de $\sim 30 \mathrm{~cm}$ de altura compuestas por millones de tubos por $\mathrm{m}^{2}$ (Jaubet al. 2011). Los arrecifes cubrieron casi por completo el área impactada por la descarga de aguas cloacales sin tratar, y se reportaron densidades de 650000 individuos $/ \mathrm{m}^{2}$ (Jaubet et al. 2011), 1465000 individuos $/ \mathrm{m}^{2}$ (Garaffo et al. 2012), y más de 1650000 individuos $/ \mathrm{m}^{2}$ (Jaubet 2013). La comunidad típica que hasta el momento había sido dominada por el ingeniero ecosistémico B. rodriguezii, fue modificada y pasó a estar dominada por los arrecifes del auto-ingeniero ecosistémico B. proboscidea (Jaubet et al. 2013; Elías et al. 2015; Llanos 2018). Su efecto fue 
observable tanto en las condiciones físicas como ecológicas del ambiente invadido, lo que afectó tanto la dinámica como la diversidad y la abundancia de todos los organismos infaunales y epifaunales de la comunidad natural dominada por el bivalvo B. rodriguezii (Jaubet et al. 2011; Jaubet et al. 2013; Sánchez 2014; Elías et al. 2015; Elías et al. 2017).

En diciembre de 2014 se inauguró oficialmente el emisario submarino (Osse 2014) (www.mardelplata.gob.ar/Noticias/ inauguraron-el-emisario). Desde entonces, la descarga de los desechos se realiza lejos de la línea de costa (a $4 \mathrm{~km}$ ). Por lo tanto, se espera que en el área intermareal aumente la abundancia de las especies sensibles a la contaminación orgánica y disminuya la abundancia de las especies oportunistas; así como también se incrementen los valores de los parámetros comunitarios: diversidad, riqueza y equitatividad.

El objetivo de este estudio fue evaluar la respuesta de la estructura de la comunidad bentónica intermareal a la construcción y la puesta en marcha de un emisario submarino en la ciudad de Mar del Plata. Para ello se consideraron tres etapas que abarcan todo el proceso de construcción y puesta en funcionamiento: Antes (etapa previa a cualquier modificación o construcción), Durante (etapa que abarca desde el momento en que se comenzó a construir las escolleras de protección hasta la finalización de la obra) y Después (fase posterior a la puesta en funcionamiento del ES).

\section{Materiales y Métodos}

\section{Toma de muestras}

Las muestras se colectaron en dos localidades de la provincia de Buenos Aires (Argentina):

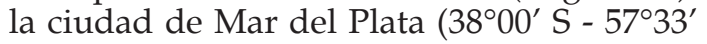
O), Partido de General Pueyrredón, y Santa

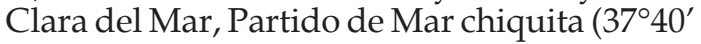
$\left.\mathrm{S}-57^{\circ} 30^{\prime} \mathrm{O}\right)$. Se consideraron tres sitios de muestreo localizados a diferentes distancias del efluente cloacal. El sitio 1 es el más cercano al punto de descarga intermareal $(1200 \mathrm{~m}$ al sur; Partido General Pueyrredón), el sitio 2 se encuentra a $8000 \mathrm{~m}$ al norte (Partido de Mar Chiquita) y el sitio 3 está localizado a $9000 \mathrm{~m}$ al norte (Partido de Mar Chiquita) (Figura 1).

Los muestreos se realizaron durante las tres etapas asociadas a la construcción del ES: febrero del 2008 ( $\mathrm{A}=$ antes; etapa anterior a

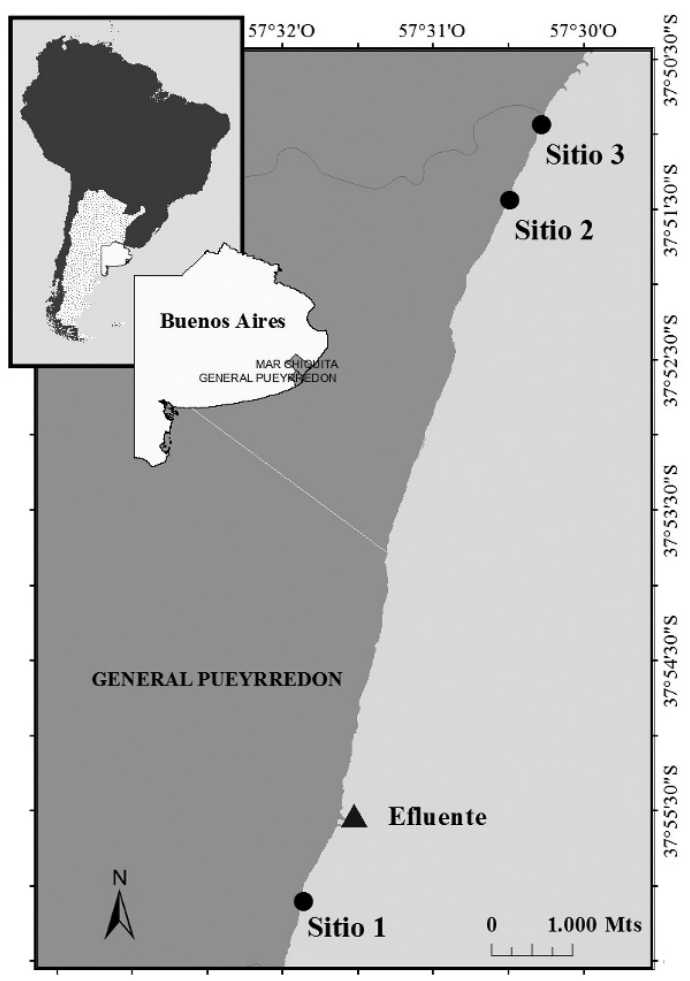

Figura 1. Mapa del área de estudio detallando los tres sitios de muestreo. El sitio 1 es el más cercano al punto de descarga intermareal (1200 m al sur, Partido de General Pueyrredón); el sitio 2 se encuentra a $8000 \mathrm{~m}$ al norte; el sitio 3 está localizado a $9000 \mathrm{~m}$ al norte (ambos pertenecientes al Partido de Mar Chiquita). Efluente: zona de descarga intermareal en las etapas Antes y Durante.

Figure 1. Map of the study area with the three sampling sites. Site 1 is the closest site to the intertidal discharge point (1200 m southward, Partido de General Pueyrredón); site 2, at $8000 \mathrm{~m}$ northward; site 3, at $9000 \mathrm{~m}$ northward (both belonging to Partido de Mar Chiquita). Effluent: intertidal discharge zone in both Before and During phases.

la construcción del emisario), febrero 2013 $(\mathrm{Du}=$ Durante; fase correspondiente a la etapa de construcción del emisario) y febrero 2015 (De=después; etapa posterior a la puesta en marcha del emisario). En cada sitio se seleccionaron tres estaciones separadas $50 \mathrm{~m}$ entre sí, en las que se tomaron tres repeticiones. En total se colectaron 9 muestras por sitio para cada etapa. Las unidades de muestreo (UM) se colectaron en forma aleatoria en rocas intermareales independientes, por medio de un cilindro plástico de PVC (core) de $78 \mathrm{~cm}^{2}$ de superficie. Además, se registraron tres mediciones de temperatura y $\mathrm{pH}$ del agua de mar en cada sitio durante cada etapa, con un equipo multiparamétrico HORIBA U-10. Para medir los niveles de materia orgánica en sedimento se tomaron 6 muestras aleatorias (30 g) en cada sitio de muestro durante cada etapa. El porcentaje de materia orgánica total 
(MOT) fue determinado por el método de calcinación (Byers et al. 1978).

Cada UM (i.e., organismos y sedimento retenido) fue conservada con agua de mar en frascos de plástico y fijadas con una solución de formaldehído (5\%) hasta su posterior análisis en el laboratorio. Cada UM se tamizó a través de dos mallas $1 \mathrm{~mm}$ y $0.5 \mathrm{~mm}$ y se conservaron en alcohol 70\% v/v. Luego los organismos retenidos se identificaron al menor nivel taxonómico posible y se cuantificaron.

\section{Análisis de datos}

Con el objetivo de caracterizar los sitios de estudio en las diferentes etapas se realizó un ANOVA de dos vías para cada una de las variables ambientales medidas $(\mathrm{pH}$, temperatura y MOT). Se analizó el efecto del sitio y etapa, y su interacción, sobre el $\mathrm{pH}$, la temperatura, la salinidad y la MOT. Previamente se corroboraron los supuestos estadísticosdenormalidadyhomocedasticidad de los datos. La normalidad fue evaluada por medio de un gráfico Q-Q y la homogeneidad de varianza fue analizada por medio de la prueba $\mathrm{C}$ de Cochran y el gráfico de residuales (Zar 1999). Se utilizó la prueba de Tukey para las comparaciones a posteriori.

Para cada UM se calcularon los parámetros comunitarios: riqueza específica (S), equitatividad de Pielou (J') (Pielou 1969) y diversidad de Shannon-Wiener $\left(\mathrm{H}^{\prime}\right)$ (Shannon and Weaver 1963). Luego, se analizaron los efectos de los factores sitio, estación (anidada en sitio) y etapa, y sus interacciones, sobre dichos parámetros comunitarios mediante el uso de modelos lineales generales. Se utilizó la prueba de Tukey para las comparaciones a posteriori. Para explorar la relación entre los parámetros comunitarios y las variables ambientales se realizó un análisis de componentes principales (ACP).

Se realizó un análisis PERMANOVA (del inglés permutational multivariate analysis of variance) para evaluar el efecto de los factores sitio y etapa sobre la composición del ensamble de especies, utilizando el método permutacional de modelo completo (Clarke and Warwick 2001; Anderson et al. 2008). Para ello, a la matriz básica de datos se le aplicó una transformación raíz cuarta de manera de minimizar el peso a las especies dominantes y se retiraron las especies que tenían menos de dos valores de presencia en toda la matriz. Luego, se calculó la matriz de similitud de acuerdo al índice de Bray-Curtis. Se realizó un análisis SIMPER (del inglés similarity percentage analysis) para evaluar el aporte relativo de las especies a la disimilaridad que hay entre los grupos (Clarke 1993). Finalmente, se realizó un ordenamiento multidimensional MDS (del inglés multidimensional scaling) con el objetivo de detectar los agrupamientos naturales de las comunidades de acuerdo a la similitud en su composición específica (Oksanen et al. 2013).

\section{Resultados}

Se encontró un total de 38 taxa, de los cuales 15 pertenecen a poliquetos (espiónidos, capitélidos, sílidos, nereidos, lumbrinéridos, entre otros) y otros 12 son artrópodos (cirripedios, anfípodos e isópodos, entre otros). También se encontraron algunas especies de moluscos (bivalvos, gasterópodos, entre otros) y en menor número nemertinos, nematodos, cnidarios, platelmintos y oligoquetos.

La temperatura varió de manera diferencial en los sitios a lo largo de las tres etapas (sitio*etapa, $\left.\mathrm{F}_{(4,32)}=6.5, P<0.01\right)$. Se detectó una disminución de la temperatura en los tres sitios a lo largo de las etapas; el sitio 2 fue el que presentó valores más bajos durante la etapa A y valores más altos durante la etapa Du (Figura 2a, Tabla 1). El pH varió de manera diferencial en los sitios a lo largo de las tres etapas (sitio*etapa, $\mathrm{F}_{(4,32)}=5.4, P<0.01$ ). En las etapas A y Du, el sitio 1 presentó valores más bajos de $\mathrm{pH}$ que el resto de los sitios. Todos los sitios presentaron un aumento en el valor del $\mathrm{pH}$ en la etapa posterior a la puesta en

\begin{tabular}{lllllllll}
\hline & S1-A & S1-Du & S1-De & S2-A & S2-Du & S2-De & S3-A & S3-Du \\
\hline S1-Du & 0.684 & & & & & & & \\
S1-De & $<0.001^{*}$ & $0.002^{*}$ & & & & & & \\
S2-A & 0.055 & 0.684 & 0.207 & & & & & \\
S2-Du & 1.000 & 0.452 & $<0.001^{*}$ & $0.019^{*}$ & & & & \\
S2-De & $<0.001^{*}$ & $<0.001^{*}$ & 0.841 & $0.006^{*}$ & $<0.001^{*}$ & & & \\
S3-A & 0.996 & 0.259 & $<0.001^{*}$ & $0.014^{*}$ & 0.997 & $<0.001^{*}$ & & \\
S3-Du & 0.508 & 1.000 & $0.003^{*}$ & 0.838 & 0.283 & $<0.001^{*}$ & 0.163 & \\
S3-De & $<0.001^{*}$ & $<0.001^{*}$ & 0.982 & $0.022^{*}$ & $<0.001^{*}$ & 1.000 & $<0.001^{*}$ & $<0.001^{*}$ \\
\hline
\end{tabular}

Tabla 1. Resultados del análisis de la prueba de Tukey (comparación a posteriori) para evaluar la interacción $\mathrm{s}^{*} \mathrm{e}$ sobre la variable temperatura.

Table 1. Results of Tukey test analysis (a posteriori) to evaluate ${ }^{*} \mathrm{e}$ interaction on temperature. 
a

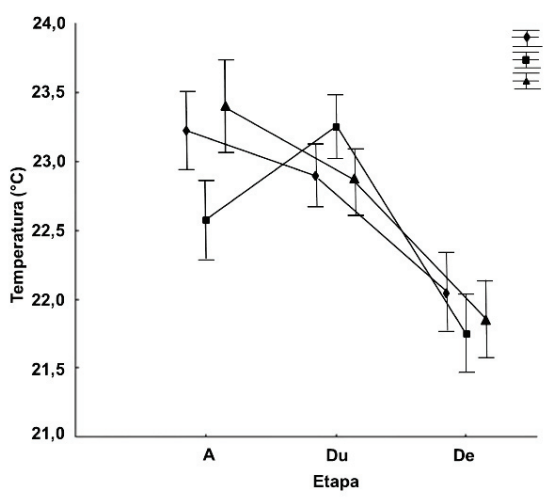

b

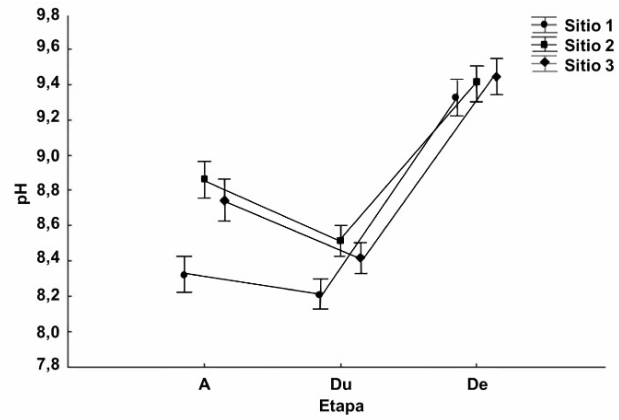

C

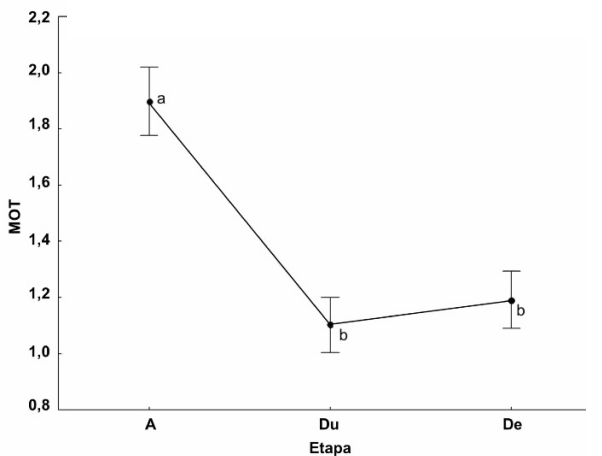

Figura 2. Variación espacio-temporal de a) temperatura, b) $\mathrm{pH}$ y c) materia orgánica total (MOT) en los sitios de estudio [sitio $1(1200 \mathrm{~m}$ al sur de efluente; Partido de General Pueyrredón), sitio 2 (8000 m al norte; Partido de Mar Chiquita) y sitio 3 (9000 $\mathrm{m}$ al norte del efluente; Partido de Mar Chiquita)], en cada etapa de estudio [antes de la construcción del emisario (A), durante la construcción $(\mathrm{Du})$ y posterior a la construcción (De)]. El punto medio indica la media y el largo de las barras el 0.95 de los intervalos de confianza. Las letras indican diferencias significativas $(P<0.01)$. Cuando la interacción $S * E$ no resultó significativa, los datos correspondientes a los sitios fueron agrupados.

Figure 2. Spatial-temporal variation of the a) temperature, b) $\mathrm{pH}$ and c) total organic matter (TOM) among the sampling sites [site 1 (1200 m southward of the effluent; Partido de General Pueyrredón), site 2 (8000 m northward; Partido de Mar de Chiquita) and site 3 (9000 m northward of the effluent; Partido de Mar Chiquita)], in each phase of the study [before the construction of the submarine outfall (A), during the construction (Du) and after the construction (De)]. The midpoint indicates the mean and the length of the bars indicates the 0.95 of the confidence intervals. The letters indicate significant differences $(P<0.01)$. When the $S^{*} E$ interaction was not significant, the sites data were grouped. a
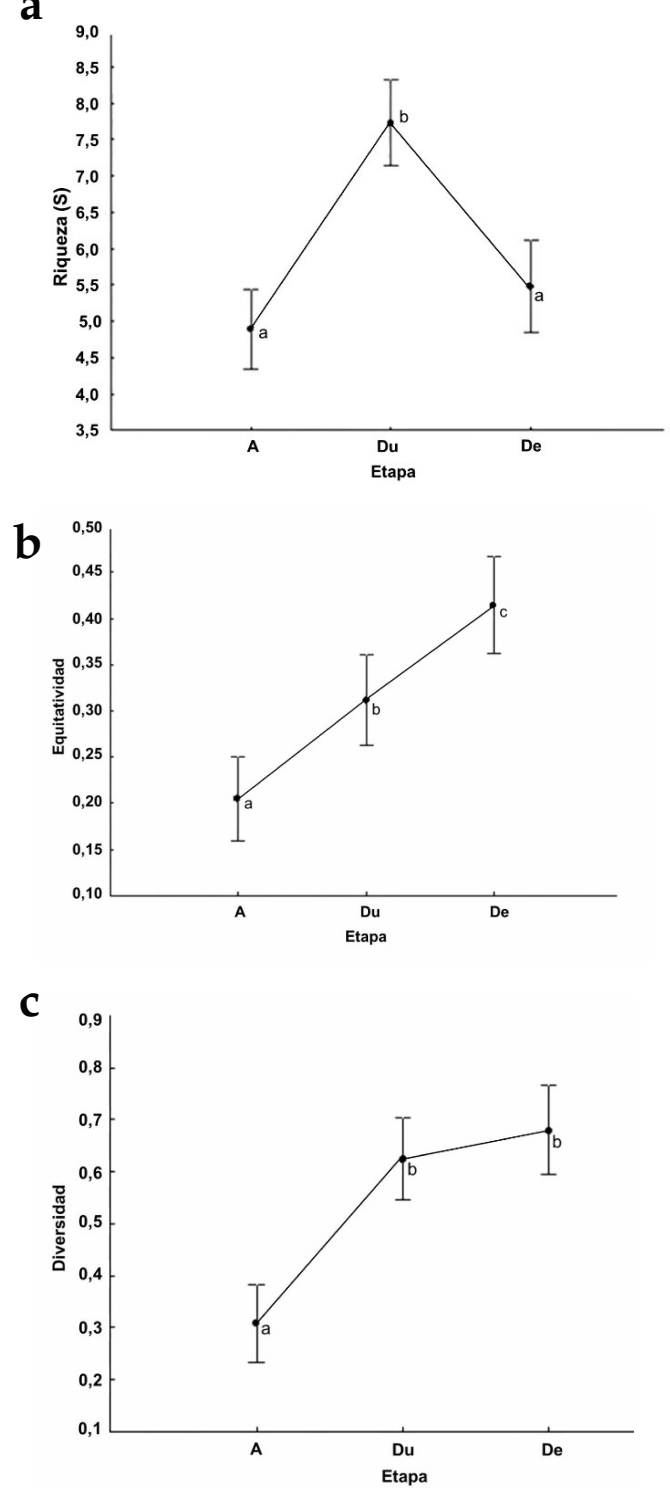

Figura 3. Variación temporal de la a) riqueza, b) equitatividad y c) diversidad en los sitios de estudio [sitio 1 (1200 m al sur de efluente), sitio 2 (8000 m al norte) y sitio 3 (9000 $\mathrm{m}$ al norte del efluente)], en las distintas etapas [antes de la construcción del emisario (A), durante la construcción $(\mathrm{Du})$ y posterior a la construcción $(\mathrm{De})]$. El punto medio indica la media y el largo de las barras el 0.95 de los intervalos de confianza. Las letras indican diferencias significativas $(P<0.01)$. Cuando la interacción $S^{*} E$ no resultó significativa, los datos correspondientes a los sitios fueron agrupados.

Figure 3. Temporal variation of: a) richness index, b) equitativy and c) diversity in the different study sites [site 1 (1200 m southward of the effluent), site 2 (8000 m northward) and site 3 (9000 m northward of the effluent)], among the different phases [before the construction of the submarine outfall $(\mathrm{A})$, during the construction $(\mathrm{Du})$ and after the construction (De)]. The midpoint indicates the mean and length of the bars at 0.95 of the confidence intervals. The letters indicate significant differences $(P<0.01)$. When the $S^{*} E$ interaction was not significant, the sites data were grouped. 
Tabla 2. Resultados del análisis de la prueba de Tukey (comparación a posteriori) para evaluar la interacción $S^{*} E$ sobre la variable $\mathrm{pH}$.

Table 2. Results of Tukey test analysis (a posteriori) to evaluate $S^{*} E$ interaction on $\mathrm{pH}$

\begin{tabular}{lllllllll}
\hline & S1-A & S1-Du & S1-De & S2-A & S2-Du & S2-De & S3-A & S3-Du \\
\hline S1-Du & 0.720 & & & & & & & \\
S1-De & $<0.001^{*}$ & $<0.001^{*}$ & & & & & & \\
S2-A & $<0.001^{*}$ & $<0.001^{*}$ & $<0.001^{*}$ & & & & & \\
S2-Du & 0.106 & $<0.001^{*}$ & $<0.001^{*}$ & 0.001 & & & & \\
S2-De & $<0.001^{*}$ & $<0.001^{*}$ & 0.984 & $<0.001^{*}$ & $<0.001^{*}$ & & & \\
S3-A & $<0.001^{*}$ & $<0.001^{*}$ & $<0.001^{*}$ & 0.843 & 0.095 & $<0.001^{*}$ & & \\
S3-Du & 0.875 & $0.029^{*}$ & $<0.001^{*}$ & $<0.001^{*}$ & 0.723 & $<0.001^{*}$ & $0.003^{*}$ & \\
S3-De & $<0.001^{*}$ & $<0.001^{*}$ & 0.775 & $<0.001^{*}$ & $<0.001^{*}$ & 0.999 & $<0.001^{*}$ & $<0.001^{*}$ \\
\hline
\end{tabular}

Tabla 3. Resultados del análisis PERMANOVA. En la primera columna se indican los factores: sitio, etapa y residuales. En la primera fila se indican los valores de g.l. (grados de libertad), SS (suma de cuadrados), MS (cuadrados medios), Pseudo-F (estadístico), P (valor de significancia), Perms (permutaciones).

Table 3. Results of the PERMANOVA analysis. The first column shows the site, phase and residuals. In the first row, d.f. (degrees of freedom), SS (sum of squares), MS (mean square), Pseudo-F (statistical), $P$ (value of significance), Perms (permutations) are indicated.

\begin{tabular}{lcccccc}
\hline Factores & d.f. & SS & MS & Pseudo-F & $P$ & Perms \\
\hline Sitio (S) & 2 & 5235.8 & 2617.9 & 4.3911 & 0.001 & 999 \\
Etapa (E) & 2 & 26535 & 13268 & 22.254 & 0.001 & 999 \\
S*E & 4 & 5214.6 & 1303.7 & 2.1867 & 0.003 & 999 \\
Residuales & 85 & 50676 & 596.19 & & & \\
Total & 93 & 87707 & & & & \\
\hline
\end{tabular}

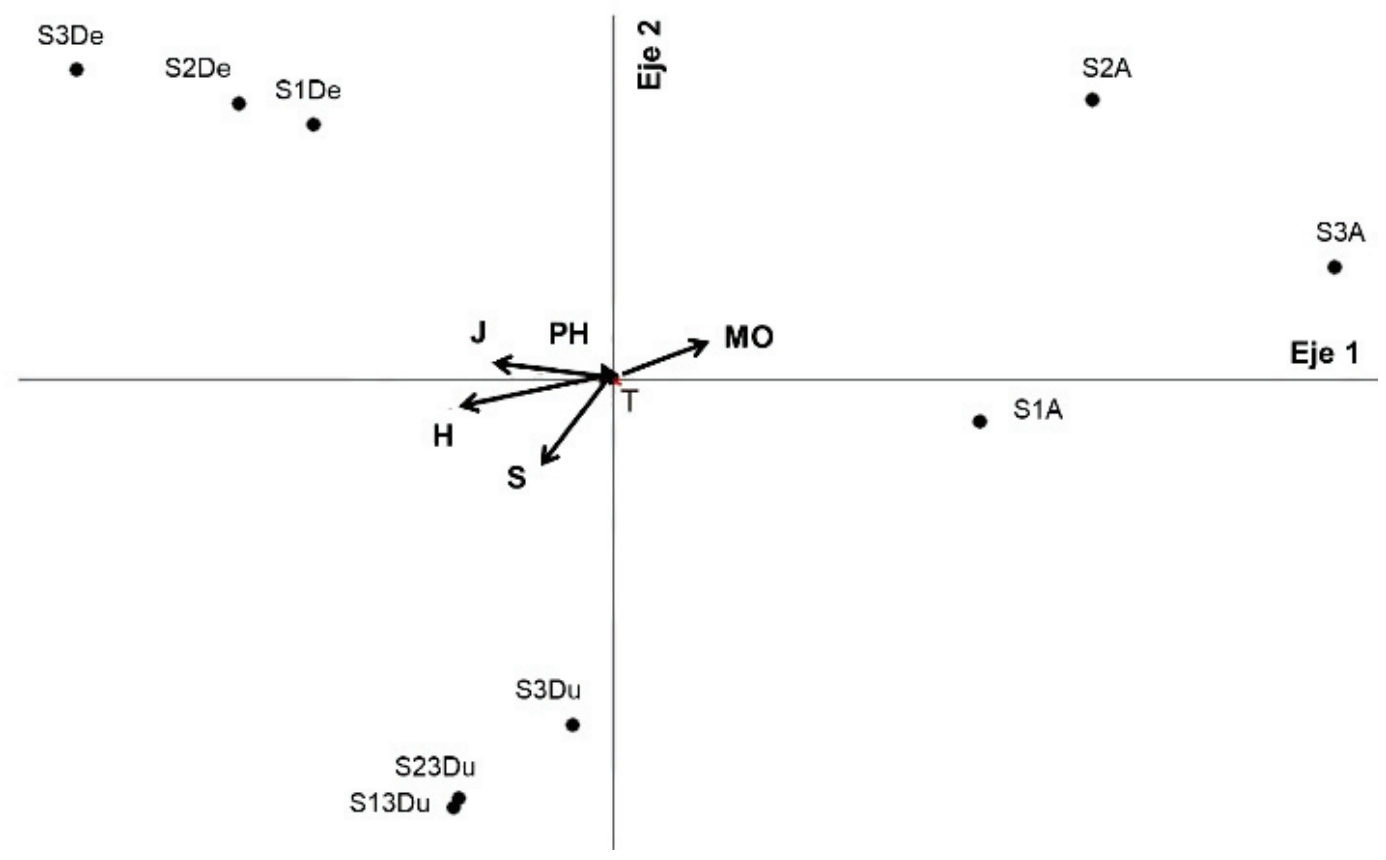

Figura 4. Análisis de componentes principales (ACP) integrando las variables biológicas (S: riqueza, J: equitatividad y H: diversidad) y las variables ambientales (T: temperatura, MOT: materia orgánica total y $\mathrm{pH}$ ) en los tres sitios de muestreo durante las diferentes etapas [antes de la construcción del emisario (A), durante la construcción (Du) y posterior a la construcción (De)].

Figure 4. Principal components analysis (PCA) integrating biological variables (S: richness, J: evenness and H: diversity) and Environmental variables (T: temperature, TOM: total organic matter and $\mathrm{pH}$ ) in the three sampling sites during the different phases [before the construction of the submarine outfall (A), during the construction (Du) and after the construction (De)]. 
funcionamiento del emisario submarino (Figura 2 b, Tabla 2). La MOT presentó una disminución marcada luego de la etapa A en todos los sitios (etapa, F=59.37, g.1.=2 y $P<0.01$ ) (Figura 2c).

La riqueza (S) varió a lo largo de las tres etapas (etapa, $\mathrm{F}=46.67$, g.1.=2 y $P<0.001$ ). En general, se observó un aumento de sus valores en la etapa Du y una disminución en las etapas A y De (Figura 3a). Los valores de equitatividad $\left(\mathrm{J}^{\prime}\right)$ presentaron un aumento marcado a lo largo del tiempo (etapa $\mathrm{F}=9.83$, g.1.=2 y $P<0.001)$. Se observaron menores valores en la etapa A y mayores valores en las etapas Du y De (Figura 3b). Con respecto a la diversidad $\left(\mathrm{H}^{\prime}\right)$, durante la etapa A se observaron valores más bajos que en el resto de las etapas (etapa F=22.64, g.l.=2 y $P<0.001$ ) (Figura 3c). No se presentaron diferencias significativas con respecto al efecto del factor sitio ni del factor estación (anidado en sitio).
A partir del análisis de componentes principales, se observa que el primer componente (eje X) explica $60.07 \%$ de la variabilidad de los datos. Las variables que contribuyeron a la formación del primer eje fueron MOT (materia orgánica total), la diversidad y la equitatividad. En este eje se observa un aumento de MOT hacia la derecha y un patrón opuesto para la equitatividad y la diversidad. Por lo tanto, en el primer componente, al aumentar MOT disminuyen los valores de equitatividad y de diversidad. De acuerdo con este eje, los tres sitios presentaron un mayor valor de MOT durante la etapa A y un menor valor de MOT durante la etapa De (Figura 4).

Según el análisis de PERMANOVA, la interacción sitio*etapa tuvo un efecto significativo sobre la composición del ensamble de especies (Tabla 3). Debido a esta interacción, se analizaron por separado

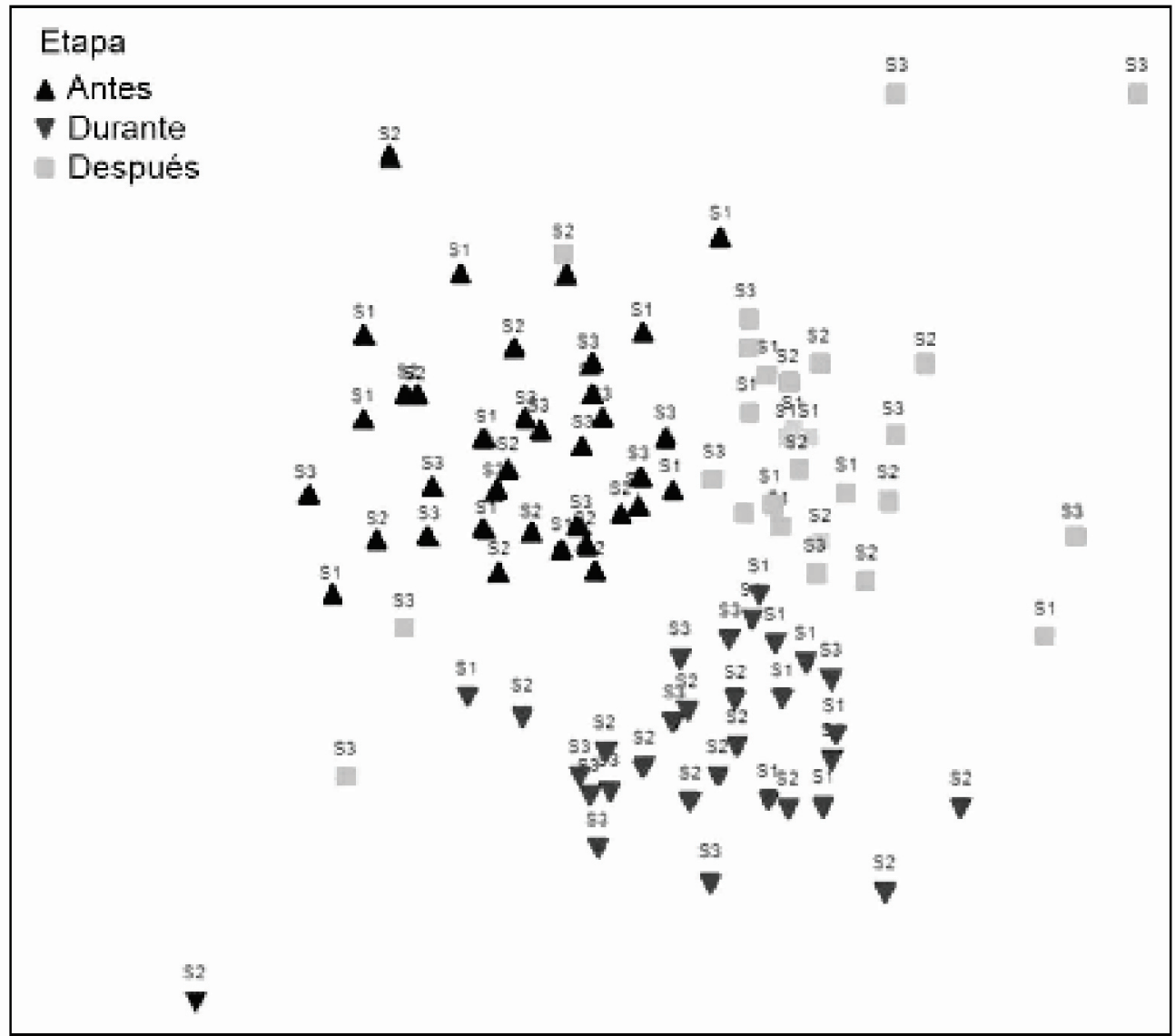

Figura 5. Gráfico de ordenamiento multidimensional MDS. Los puntos representan los agrupamientos de las especies dentro de cada sitio [sitio 1 (1200 m al sur de efluente), sitio 2 (8000 m al norte) y sitio 3 (9000 m al norte del efluente)], ordenados en el espacio multidimensional en las distintas etapas. Stress $(=0,2)$, indica una buena representación en dimensiones reducidas.

Figure 5. MDS graph. Points represent the groupings of species within each site [site 1 (1200 m southward of the effluent), site 2 (8000 m northward) and site 3 (9000 m northward of the effluent)] arranged in the multidimensional space at the different phases. Stress $(=0.2)$; indicate a good representation in reduced dimensions. 
Tabla 4. Análisis a posteriori de la prueba pair-wise y análisis SIMPER para aquellos pares de grupos en los que hubo diferencias de acuerdo a su composición de especies. Prom. Abund. (abundancia promedio), Acum. \% (porcentaje de contribución acumulada)

Table 4. A posteriori analysis of pair-wise test and SIMPER analysis for those pairs of groups in which there were differences according to their species composition. Prom. Abund. (average abundance), Acum. \% (percentage of cumulated contribution).

\begin{tabular}{|c|c|c|c|c|c|c|c|c|}
\hline \multicolumn{3}{|c|}{ Prueba pair-wise } & \multirow[b]{2}{*}{$\begin{array}{c}\text { Disimilaridad } \\
(\%)\end{array}$} & \multirow{2}{*}{$\begin{array}{l}\text { SIMPER } \\
\text { Especie }\end{array}$} & \multicolumn{2}{|c|}{ Etapas } & \multirow[b]{2}{*}{$\begin{array}{c}\text { Contribución } \\
\%\end{array}$} & \multirow[b]{2}{*}{$\begin{array}{c}\text { Acum. } \\
\%\end{array}$} \\
\hline & $\mathrm{T}$ & $P$ & & & $\begin{array}{l}\text { Prom. } \\
\text { Abund. }\end{array}$ & $\begin{array}{l}\text { Prom. } \\
\text { Abund. }\end{array}$ & & \\
\hline \multicolumn{9}{|c|}{ 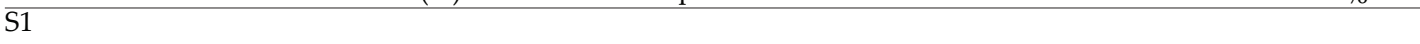 } \\
\hline \multirow[t]{17}{*}{$\mathrm{A} \neq \mathrm{Du}$} & 3.336 & 0.001 & 44.47 & B. proboscidea & 1.71 & 3.59 & 16.14 & 16.14 \\
\hline & & & & S. Prolixa & 1.11 & 2.32 & 10.29 & 26.43 \\
\hline & & & & M. edulis & 0.53 & 1.52 & 7.46 & 33.89 \\
\hline & & & & Notoplana sp. & 0 & 1.1 & 7.45 & 41.34 \\
\hline & & & & Actiniaria & 0 & 1.1 & 7.45 & 48.79 \\
\hline & & & & S. lessoni & 0.9 & 1.72 & 7.36 & 56.14 \\
\hline & & & & B. rodriguezii & 4.34 & 5.3 & 7.02 & 63.17 \\
\hline & & & & Phylum Nemertea & 0.08 & 0.78 & 5.18 & 68.35 \\
\hline & & & & Syllidae sp. & 1.3 & 1.63 & 4.58 & 72.93 \\
\hline & & & & Familia Nereididae & 0 & 0.57 & 3.78 & 76.71 \\
\hline & & & & L. bonaerensis & 0.36 & 0.2 & 3.12 & 79.83 \\
\hline & & & & Familia Lumbrineridae & 0.35 & 0 & 2.23 & 82.06 \\
\hline & & & & Megalopa cangrejo & 0 & 0.33 & 2.15 & 84.21 \\
\hline & & & & B. glandula & 0 & 0.3 & 2.12 & 86.33 \\
\hline & & & & H. grandicornis & 0 & 0.23 & 1.57 & 87.9 \\
\hline & & & & Monocorophyum sp. & 0 & 0.2 & 1.5 & 89.4 \\
\hline & 2746 & 0001 & 4199 & J. falcata & 0 & 0.22 & 1.42 & 90.82 \\
\hline \multirow{11}{*}{$\mathrm{A} \neq \mathrm{De}$} & 2.146 & 0.001 & 41.99 & S. Prolixa. & 1.11 & 2.66 & 16.23 & 16.23 \\
\hline & & & & Notoplana sp. & 0 & 1.71 & 16.09 & 32.32 \\
\hline & & & & B. proboscidea. & 1.71 & 1.51 & 15.34 & 47.66 \\
\hline & & & & S. lessoni & 0.9 & 0.94 & 8.29 & 55.96 \\
\hline & & & & Syllidae sp. & 1.3 & 1.57 & 6.64 & 62.6 \\
\hline & & & & B. rodriguezii & 4.34 & 4.4 & 5.65 & 68.24 \\
\hline & & & & L. bonaerensis & 0.36 & 0.38 & 5.14 & 73.38 \\
\hline & & & & M. edulis & 0.53 & 0 & 4.72 & 78.09 \\
\hline & & & & H. grandicornis & 0 & 0.49 & 4.43 & 82.52 \\
\hline & & & & P. Nemertea & 0.08 & 0.38 & 3.6 & 86.12 \\
\hline & & & & F. Nereididae & 0 & 0.35 & 3.17 & 89.29 \\
\hline \multirow{19}{*}{$\mathrm{Du} \neq \mathrm{De}$} & 2666 & 0.001 & 3868 & L. tetraura & 0.35 & 0 & 2.97 & 92.26 \\
\hline & 2.066 & 0.001 & 38.68 & B. proboscidea & 3.59 & 1.51 & 18.35 & 18.35 \\
\hline & & & & M. edulis & 1.52 & 0 & 10.38 & 28.73 \\
\hline & & & & Actinaria & 1.1 & 0 & 7.59 & 36.32 \\
\hline & & & & S. lessoni & 1.72 & 0.94 & 7.17 & 43.49 \\
\hline & & & & B. rodriguezii & 5.3 & 4.4 & 6.6 & 50.09 \\
\hline & & & & Syllidae sp. & 1.63 & 1.57 & 5.34 & 55.43 \\
\hline & & & & S. prolixa & 2.32 & 2.66 & 5.05 & 60.48 \\
\hline & & & & P. Nemertea & 0.78 & 0.38 & 5.03 & 65.51 \\
\hline & & & & Notoplana sp. & 1.1 & 1.71 & 4.51 & 70.02 \\
\hline & & & & F. Nereididae & 0.57 & 0.35 & 4 & 74.02 \\
\hline & & & & H. grandicornis & 0.23 & 0.49 & 3.69 & 77.7 \\
\hline & & & & L. bonaerensis & 0.2 & 0.38 & 3.11 & 80.82 \\
\hline & & & & Megalopa cangrejo & 0.33 & 0 & 2.2 & 83.02 \\
\hline & & & & B. glandula & 0.3 & 0 & 2.15 & 85.16 \\
\hline & & & & F. Lumbrinelidae & 0 & 0.24 & 1.68 & 86.84 \\
\hline & & & & Monocorophyum sp. & 0.2 & 0 & 1.51 & 88.36 \\
\hline & & & & J. falcata & 0.22 & 0 & 1.46 & 89.81 \\
\hline & & & & Megalopa cangrejo & 0.2 & 0 & 1.44 & 91.25 \\
\hline
\end{tabular}


Tabla 4. Continuación.

Table 4. Continuation.

\begin{tabular}{|c|c|c|c|c|c|c|c|c|}
\hline \multicolumn{9}{|l|}{ S2 } \\
\hline \multirow[t]{17}{*}{$\mathrm{A} \neq \mathrm{Du}$} & \multirow[t]{17}{*}{3.373} & \multirow[t]{17}{*}{0.001} & \multirow[t]{17}{*}{48.03} & S. lessoni & 1.23 & 2.83 & 11.55 & 11.55 \\
\hline & & & & B. proboscidea & 1.83 & 2.19 & 9.78 & 21.33 \\
\hline & & & & H. grandicornis & 0 & 1.12 & 7.81 & 29.14 \\
\hline & & & & S. prolixa & 0.46 & 1.4 & 6.65 & 35.79 \\
\hline & & & & B. rodriguezii & 4.76 & 5.11 & 6.4 & 42.2 \\
\hline & & & & Syllidae sp. & 1.62 & 2.11 & 5.42 & 47.62 \\
\hline & & & & P. Nemertea & 0 & 0.86 & 5.07 & 52.69 \\
\hline & & & & B. glandula & 0 & 0.87 & 4.89 & 57.59 \\
\hline & & & & Orden Harpacticoida & 0 & 077 & 4.8 & 62.39 \\
\hline & & & & Notoplana sp & 0 & 0.76 & 4.44 & 66.83 \\
\hline & & & & M. edulis & 0.38 & 0.69 & 3.91 & 70.74 \\
\hline & & & & Cyrtograpsus altimanus & 0 & 0.54 & 3.25 & 73.99 \\
\hline & & & & R. glútea & 0 & 0.48 & 3.09 & 77.08 \\
\hline & & & & Megalopa cangrejo & 0 & 0.43 & 3.06 & 80.14 \\
\hline & & & & F. Nereididae & 0 & 0.53 & 3.04 & 83.17 \\
\hline & & & & L. bonaerensis & 0.44 & 0.1 & 2.86 & 86.03 \\
\hline & & & & I. báltica & 0.08 & 0.41 & 2.84 & 88.87 \\
\hline \multirow{12}{*}{$\mathrm{A} \neq \mathrm{De}$} & \multirow{12}{*}{3.116} & \multirow{12}{*}{0.001} & \multirow{12}{*}{44.56} & S. serratum & 0 & 0.28 & 1.91 & 90.78 \\
\hline & & & & S. prolixa & 0.46 & 2.61 & 18.68 & 18.68 \\
\hline & & & & B. proboscidea & 1.83 & 0.78 & 12.6 & 31.28 \\
\hline & & & & Notoplana sp. & 0 & 1.14 & 9.38 & 40.66 \\
\hline & & & & S. lessoni & 1.23 & 1.92 & 9.25 & 49.91 \\
\hline & & & & B. rodriguezii & 4.76 & 4.53 & 8.04 & 57.96 \\
\hline & & & & H. grandicornis & 0 & 0.92 & 7.88 & 65.84 \\
\hline & & & & Syllidae sp. & 1.62 & 1.16 & 5.85 & 71.69 \\
\hline & & & & L. bonaerensis & 0.44 & 0.44 & 5.04 & 76.73 \\
\hline & & & & I. báltica & 0.08 & 0.59 & 4.86 & 81.59 \\
\hline & & & & Orden Calanoida & 0 & 0.38 & 3.47 & 85.06 \\
\hline & & & & M. edulis & 0.38 & 0 & 2.89 & 87.95 \\
\hline \multirow{21}{*}{ DufDe } & \multirow{21}{*}{2.499} & \multirow{21}{*}{0.001} & \multirow{21}{*}{46.75} & F. Phyllodocidae & 0 & 0.35 & 2.76 & 90.71 \\
\hline & & & & B. proboscidea & 2.19 & 0.78 & 8.78 & 8.78 \\
\hline & & & & S. prolixa & 1.4 & 2.61 & 8.37 & 17.15 \\
\hline & & & & S. lessoni & 2.83 & 1.92 & 7.85 & 25 \\
\hline & & & & B. rodriguezii & 5.11 & 4.53 & 7.28 & 32.28 \\
\hline & & & & Syllidae sp. & 2.11 & 1.16 & 6.48 & 38.76 \\
\hline & & & & H. grandicornis & 1.12 & 0.92 & 5.2 & 43.96 \\
\hline & & & & Notoplana sp & 0.76 & 1.14 & 5.05 & 49.01 \\
\hline & & & & B. glandula & 0.87 & 0 & 4.42 & 53.43 \\
\hline & & & & P. Nemertea & 0.86 & 0.11 & 4.41 & 57.84 \\
\hline & & & & Orden Harpacticoida & 0.77 & 0 & 4.28 & 62.12 \\
\hline & & & & I. báltica & 0.41 & 0.59 & 3.72 & 65.84 \\
\hline & & & & M. edulis & 0.69 & 0 & 3.54 & 69.37 \\
\hline & & & & Cyrtograpsus altimanus & 0.54 & 0 & 2.91 & 72.29 \\
\hline & & & & F. Nereididae & 0.53 & 0.11 & 2.79 & 75.08 \\
\hline & & & & R. glutea & 0.48 & 0 & 2.72 & 77.8 \\
\hline & & & & Megalopa cangrejo & 0.43 & 0.11 & 2.72 & 80.52 \\
\hline & & & & L. bonaerensis & 0.1 & 0.44 & 2.5 & 83.01 \\
\hline & & & & S. serratum & 0.28 & 0.24 & 2.41 & 85.42 \\
\hline & & & & Orden Calanoida & 0 & 0.38 & 2.25 & 87.67 \\
\hline & & & & F. Phyllodocidae & 0 & 0.35 & 1.88 & 89.54 \\
\hline
\end{tabular}


Tabla 4. Continuación.

Table 4. Continuation.

\begin{tabular}{|c|c|c|c|c|c|c|c|c|}
\hline \multicolumn{9}{|l|}{$\overline{\mathrm{S} 3}$} \\
\hline \multirow[t]{14}{*}{$\mathrm{A} \neq \mathrm{Du}$} & \multirow[t]{14}{*}{4.061} & \multirow[t]{14}{*}{0.001} & \multirow[t]{14}{*}{41.99} & H. grandicornis & 0 & 2.27 & 16.15 & 16.15 \\
\hline & & & & S. lesson & 1.48 & 3.02 & 11.39 & 27.53 \\
\hline & & & & B. proboscidea & 1.27 & 2.31 & 8.65 & 36.18 \\
\hline & & & & Syllidae sp. & 0.99 & 1.52 & 6.73 & 42.91 \\
\hline & & & & M. edulis & 0.24 & 1.04 & 6.71 & 49.63 \\
\hline & & & & S. prolixa & 0.94 & 1.26 & 6.22 & 55.85 \\
\hline & & & & F. Nereididae & 0 & 0.86 & 6.17 & 62.02 \\
\hline & & & & Notoplana sp & 0 & 0.84 & 5.88 & 67.91 \\
\hline & & & & S. serratum & 0.08 & 0.76 & 5.27 & 73.17 \\
\hline & & & & L. bonaerensis & 0.76 & 0.22 & 4.94 & 78.11 \\
\hline & & & & B. rodriguezii & 5.34 & 5.74 & 4.06 & 82.17 \\
\hline & & & & B. glandula & 0 & 0.37 & 2.75 & 84.92 \\
\hline & & & & Cyrtograpsus altimanus & 0 & 0.32 & 2.19 & 87.1 \\
\hline & & & & Megalopa cangrejo & 0 & 0.27 & 1.78 & 88.89 \\
\hline \multirow{12}{*}{$\mathrm{A} \neq \mathrm{De}$} & \multirow{12}{*}{2.747} & \multirow{12}{*}{0.001} & \multirow{12}{*}{47.77} & O. Tanaidacea & 0 & 0.22 & 1.65 & 90.54 \\
\hline & & & & B. rodriguezzi & 5.34 & 3.68 & 15.94 & 15.94 \\
\hline & & & & S. prolixa & 0.94 & 1.82 & 11.77 & 27.71 \\
\hline & & & & B. proboscidea & 1.27 & 0.91 & 11.33 & 39.04 \\
\hline & & & & H. grandicornis & 0 & 1.11 & 9.57 & 48.61 \\
\hline & & & & Syllidae sp. & 0.99 & 1.22 & 8.57 & 57.18 \\
\hline & & & & S. lesson & 1.48 & 1.3 & 7.61 & 64.79 \\
\hline & & & & P. Nemertea & 0 & 0.79 & 6.44 & 71.23 \\
\hline & & & & L. bonaerensis & 0.76 & 0.11 & 6.11 & 77.34 \\
\hline & & & & S. serratum & 0.08 & 0.47 & 4.37 & 81.71 \\
\hline & & & & Notoplana sp & 0 & 0.58 & 4.21 & 85.92 \\
\hline & & & & C. dilatata & 0 & 0.29 & 2.67 & 88.59 \\
\hline \multirow{18}{*}{ Du $\neq$ De } & \multirow{18}{*}{2.527} & \multirow{18}{*}{0.001} & \multirow{18}{*}{47.89} & I. báltica & 0 & 0.35 & 2.65 & 91.23 \\
\hline & & & & B. rodriguezzi & 5.74 & 3.68 & 12.92 & 12.92 \\
\hline & & & & S. lessoni & 3.02 & 1.3 & 10.71 & 23.63 \\
\hline & & & & B. proboscidea & 2.31 & 0.91 & 10.71 & 34.34 \\
\hline & & & & S. prolixa & 1.26 & 1.82 & 7.3 & 41.63 \\
\hline & & & & H. grandicornis & 2.27 & 1.11 & 6.99 & 48.62 \\
\hline & & & & Syllidae sp. & 1.52 & 1.22 & 6.56 & 55.18 \\
\hline & & & & M. edulis & 1.04 & 0 & 6.13 & 61.31 \\
\hline & & & & Notoplana sp. & 0.84 & 0.58 & 5.11 & 66.43 \\
\hline & & & & F. Nereididae & 0.86 & 0.15 & 4.95 & 71.38 \\
\hline & & & & P. Nemertea & 0.22 & 0.79 & 4.72 & 76.1 \\
\hline & & & & S. serratum & 0.76 & 0.47 & 3.67 & 79.77 \\
\hline & & & & B. glandula & 0.37 & 0 & 2.29 & 82.06 \\
\hline & & & & I. báltica & 0.11 & 0.35 & 2.14 & 84.2 \\
\hline & & & & Cyrtograpsus altimanus & 0.32 & 0 & 1.83 & 86.03 \\
\hline & & & & C. dilatata & 0 & 0.29 & 1.78 & 87.81 \\
\hline & & & & L. bonaerensis & 0.22 & 0.11 & 1.67 & 89.47 \\
\hline & & & & Megalopa cangrejo & 0.27 & 0 & 1.49 & 90.97 \\
\hline
\end{tabular}

las variaciones entre etapas para cada sitio en particular. Estas comparaciones pareadas evidenciaron variaciones temporales (etapas) (Tabla 4).

El análisis SIMPER mostró que los aportes individuales de las especies a la diferenciación entre grupos fueron menores a $20 \%$. Con respecto a la variación temporal, en cada uno de los sitios se encontraron diferencias entre todas las etapas. En el sitio 1, las diferencias entre las etapas A y Du estuvieron dadas principalmente por las especies de poliquetos Boccardia proboscidea y Syllis prolixa. Ambas especies presentaron una mayor abundancia en la etapa Du. Las diferencias entre A y De 
estuvieron dadas principalmente por S. prolixa, Notoplana sp. y B. proboscidea. Las dos primeras presentaron mayor abundancia en la etapa De, y B. proboscidea, en la etapa A. Finalmente, las diferencias entre las etapas Du y De estuvieron dadas por las especies $B$. proboscidea y $M$. edulis. Las dos especies presentaron mayor abundancia en la etapa Du.

En el sitio 2, las especies que aportaron mayor contribución a las diferencias entre los ensambles de las etapas A y Du fueron la lapa Siphonaria lessoni y B. proboscidea. Ambas especies presentaron una mayor abundancia en la etapa Du. Las diferencias entre A y De estuvieron dadas mayormente por las especies S. prolixa, B. proboscidea, Notoplana sp. y $S$. lessoni. Todas presentaron un aumento en la abundancia durante la etapa De, a excepción de $B$. proboscidea, que presentó una disminución de su abundancia. B. proboscidea y S. lessoni disminuyeron su abundancia en la etapa De con respecto a la etapa Du, y S. prolixa presentó un patrón opuesto al anterior.

En el sitio 3, Hyale grandicornis, S. lessoni y B. proboscidea presentaron una mayor abundancia en la etapa Du respecto a la etapa A. Las diferencias entre las etapas A y De con respecto al ensamble de especies estuvieron dadas principalmente $(50 \%)$ por las abundancias de $B$. rodriguezii, B. proboscidea, S. prolixa y $H$. grandicornis. Las dos primeras especies disminuyeron su abundancia durante la etapa De, y las dos últimas presentaron un patrón opuesto. Finalmente, al comparar las etapas Du y De, las especies que más contribuyeron a diferenciar los ensambles fueron $B$. rodriguezii, S. lessoni y B. proboscidea. Las tres especies presentaron menos abundancia en la etapa De.

En el espacio multidimensional MDS se observan agrupamientos de acuerdo a la similitud en composición específica, correspondientes al factor etapa. Sin embargo, no se observan agrupamientos con respecto a los sitios (stress=0.2) (Figura 5).

\section{DisCUSIÓN}

Este trabajo presenta los primeros resultados acerca del efecto de la construcción y el funcionamiento del emisario submarino en Mar del Plata (Argentina). Los resultados obtenidos en los análisis de las variables ambientales evidenciaron diferencias en la calidad del agua entre los 3 periodos muestreados (antes, Durante y Después) y en los 3 sitios. En cuanto a los parámetros comunitarios, no se detectaron diferencias entre los sitios en cada una de las etapas. Una explicación posible para estos resultados podría ser el alcance que tenía la pluma de descarga, la cual provocaba que, a pesar de la distancia, los tres sitios presentaran niveles similares de impacto. En verano, la extensión de la pluma de descarga era mayor, ya que su volumen aumentaba notablemente por el incremento poblacional en Mar del Plata debido al turismo (de $2.8 \mathrm{~m}^{3} / \mathrm{s}$ en invierno a $3.5 \mathrm{~m}^{3} / \mathrm{s}$ en verano) (Scagliola et al. 2006), por lo que podía llegar a sitios más alejados. En consecuencia, durante la estación estival, los tres sitios de estudio estarían afectados de igual manera por la descarga. No haber detectado diferencias entre sitios limita parcialmente las conclusiones que se extraen de los resultados del presente estudio, ya que la falta de un sitio control impide inferir que los cambios temporales observados se deben al efecto de la construcción del emisario.

En general, los parámetros comunitarios presentaron valores más bajos en la etapa Antes, lo que pone en evidencia una mejoría durante la última etapa. En la etapa Durante se observó un aumento de la riqueza, la equitatividad y la diversidad. Esto podría estar asociado al disturbio generado en ese momento por la construcción del emisario, que al incorporar dos escolleras de abrigo y el frente de atraque en la zona pudo ocasionar un gran movimiento de la dinámica sedimentaria y dar lugar, probablemente, a nuevos espacios disponibles. Estos cambios en el ambiente pudieron propiciar el desarrollo de especies oportunistas (e.g., B. proboscidea) que se caracterizan por ser las primeras en colonizar el sustrato en las etapas iniciales de una sucesión ecológica, luego de un disturbio (Dauer and Simon 1976; Zajac and Whitlatch 1982). Según los valores de los parámetros comunitarios en la etapa Después, se podría inferir una mejoría en la calidad ambiental. Luego de la construcción del emisario, las condiciones ambientales comenzarían a mejorar (en términos de niveles de materia orgánica, temperatura y pH), por lo que la abundancia de las especies oportunistas disminuiría (B. proboscidea) y las especies nativas (B. rodriguezii) o especies con tolerancia intermedia (como por ejemplo Syllis prolixa y Notoplana sp.) volverían a colonizar el área.

Las diferencias en la composición de los ensambles de especies entre las diferentes etapas para cada sitio en particular se 
debieron principalmente a la diferencia en las abundancias relativas de hasta 20 especies o taxa. Durante las etapas Antes y Durante, la abundancia relativa de Boccardia proboscidea fue más alta con respecto a la etapa Después. Además, en el sitio 1 (1200 $\mathrm{m}$ al sur de efluente), la abundancia de esta especie presentó valores de un orden de magnitud mayor que en el resto de los sitios. La abundancia relativa de $B$. proboscidea disminuyó al aumentar la distancia al efluente intermareal. Este patrón estaría asociado con la cantidad de materia orgánica disponible. La presencia del platelminto Notoplana sp. en las etapas Durante y Después en los sitios 1 (1200 $\mathrm{m}$ al sur de efluente) y 2 (8000 $\mathrm{m}$ al norte), con mayor abundancia en la etapa posterior a la puesta en marcha del emisario submarino, se registró por primera vez en este estudio. Si bien esta especie cuenta sólo con registros de presencia en estudios anteriores (Sánchez 2014), no fue citada con densidades tan altas como las encontradas en este estudio (máximo de 3462 individuos $/ \mathrm{m}^{2}$ ). La especie podría ser indicadora de buena calidad ambiental (comunicación personal Dra. Damborenea; Museo de La Plata). En regiones tropicales, algunas especies de platelmintos (policládidos) fueron consideradas bioindicadoras de la salud de los arrecifes de coral (Quiroga et al. 2004). Sin embargo, en el Atlántico Sudoccidental aún no existen estudios que confirmen a los policládidos como indicadores de salud ambiental. Un patrón similar al encontrado con Notoplana sp. presentó la especie Syllis prolixa, cuya abundancia fue mayor en las etapas Durante y Después. La presencia de esta especie también podría estar indicando una mejora en cuanto a la salud ambiental. En general, los sílidos son muy sensibles a la contaminación: en sitios enriquecidos o impactados orgánicamente disminuyen el número de especies, de individuos o incluso desaparecen por completo (Giangrande et al. 2004; Musco et al. 2004; Sánchez et al. 2013).

El estudio de los indicadores ambientales (en agua y sedimento) y biológicos (bentónicos) en las tres etapas del proceso de construcción y puesta en marcha del ES fueron útiles para demostrar el efecto positivo que éste tiene sobre la salud ambiental. Si bien este estudio estuvo acotado a un período de tiempo corto, el conocimiento de esta dinámica abre el camino para nuevos estudios de largo plazo en donde la respuesta de los organismos bentónicos brindará herramientas aptas para el manejo costero integrado.

AgradecimiEntos . Este trabajo fue realizado con fondos presupuestarios otorgados por la Agencia Nacional de Promoción Científica y Tecnológica ANPCyT en el marco de dos PICT del FONCyT (PICT 2446- PICT 3484) y del proyecto EXA 768/16 otorgado por la Universidad Nacional de Mar del Plata. Los autores agradecen al Dr. Elías R. por el asesoramiento en el diseño del muestreo y lectura crítica del manuscrito. También agradecen a los revisores anónimos por sus valiosas correcciones y sugerencias.

\section{REFERENCIAS}

Anderson, M. J., R. N. Gorley, and K. R. Clarke. 2008. PERMANOVA+ for PRIMER: Guide to Software and Statistical Methods. PRIMER-E, Plymouth, UK.

Barañao P. A., and L. A. Tapia. 2004. Tratamiento de Aguas Servidas: Situación en Chile. Ciencia y Trabajo 13:111117.

Byers, S., C. Mills, and P. Stewart. 1978. Comparison of methods of determining organic carbon in marine sediments, with suggestions for a standard method. Hydrobiologia 58(1):43-47.

Clarke, K. R. 1993. Non-parametric multivariate analysis of changes in community structure. Australian Journal of Ecology 18:117-143.

Clarke, K. R., and R. M. Warwick. 2001. Change in Marine Communities: An Approach to Statistical Analysis and Interpretation. 2nd edition. PRIMER-E, Plymouth.

Dauer, D. M., and J. L. Simon. 1976. Habitat expansion among polychaetous annelids repopulating a defaunated marine habitat. Marine Biology 37:169-177.

Elías, R., M. S. Rivero, and E. A. Vallarino. 2003. Sewage impact on the composition and distribution of Polychaeta associated to intertidal mussel beds of the Mar del Plata rocky shore, Argentina. Iheringia, Serie Zoología 93(3): 309-318.

Elías, R., M. S. Rivero, J. R. Palacios, and E. A. Vallarino. 2006. Sewage-induced disturbance on Polychaetes inhabiting intertidal mussel beds of Brachidontes rodriguezii off Mar del Plata (Southwestern Atlantic, Argentina). En R. Sarda, G. San Martin, E. López, D. Martin and D. George (eds.). Scientific Advances on Polychaete Research. Scientia Marina 70:187-196.

Elías, R., M. A. Sánchez, M. L. Jaubet, M. S. Rivero, and E. A. Vallarino. 2009. Do treatment sewage plants really work? The intertidal mussels ${ }^{\prime \prime}$ community of the Southwestern Atlantic shore $\left(38^{\circ} \mathrm{S}, 57^{\circ} \mathrm{W}\right)$ as a case study. Revista Biología Marina y Oceanografía 44:357-368.

Elías, R., M. L. Jaubet, E. N. Llanos, M. A. Sánchez, M. S. Rivero, G. V. Garaffo, and L. Sandrini-Neto. 2015. Effect of the invader Boccardia proboscidea (Polychaete: Spionidae) on the richness, diversity, and structure of the SW Atlantic epilithic intertidal community. Marine Pollution Bulletin 91:530-536. 
Elías, R., M. L. Jaubet, A. Ferrando, and M. A. Saracho Bottero. 2017. Historia y perspectivas de los estudios sobre poliquetos en Argentina. Pp. 149 en O. Díaz-Díaz, D. Bone, C. T. Rodríguez and V. H. Delgado-Blas (eds.). Poliquetos de Sudamérica. Volumen especial del Boletín del Instituto Oceanográfico de Venezuela. Cumaná, Venezuela.

Garaffo, G. V., M. L. Jaubet, M. A. Sánchez, M. S. Rivero, E. A. Vallarino, and R. Elías. 2012. Sewage-induced polychaetes reefs in a SW Atlantic Shore: rapid response to small scale disturbance. Marine Ecology: an Evolutionary Perspesctive 33:272-279.

Giangrande, A., A. L. Delos, L. Musco, M. Licciano, and C. Pierri. 2004. Polychaete

assemblages of rocky shore along the South Adriatic coast (Mediterranean Sea). Cahiers de Biologie Marine 45:8595.

Hartman, O. 1941. Polychaetous annelids. Part III. Spionidae. Some contributions to the biology and life story of Spionidae from California. Allan Hancock Pacific Expeditions 7:289-324, pls. 45-58.

Jaubet, M. L. 2013. Boccardia proboscidea, un poliqueto invasor en el Atlántico Sudoccidental y su efecto sobre la comunidad bentónica intermareal. Tesis Doctoral, Universidad Nacional de Mar del Plata, Facultad de Ciencias Exactas y Naturales, Mar del Plata, Argentina. Pp. 207.

Jaubet, M. L., M. A. Sánchez, M. S. Rivero, G. V. Garaffo, E. A. Vallarino, and R. Elías. 2011. Intertidal biogenic reefs built by the polychaete Boccardia proboscidea in sewage-impacted areas of Argentina, SW Atlantic. Marine Ecology: an evolutionary perspective 32:188-197.

Jaubet, M. L., G. V. Garaffo, M. A. Sánchez, and R. Elías. 2013. Reef-forming polychaetes outcompetes ecosystem engineering mussels. Marine Pollution Bulletin 71:216-221.

Llanos, E. N. 2018. Biodiversidad y patrones sucesionales en comunidades bentónicas intermareales de Mar del Plata afectadas por contaminación orgánica. Tesis Doctoral, Universidad Nacional de Mar del Plata, Buenos Aires, Argentina. Pp. 219.

Madera, C. A., J. P. Silva, and M. R. Peña. 2005. Sistemas combinados para el tratamiento de aguas residuales basados en tanque séptico- filtro anaerobio y humedales superficiales. Escuela de Ingeniería de Recursos Naturales y del ambiente (EIDEMAR), Facultad de Ingeniería, Universidad del Valle. Cali, Colombia. Ingeniería y Competitividad 7:5-10.

Montera, C. 2012. Agua potable y saneamiento en la metrópoli de Buenos Aires (1993-2011) ¿Hacia una superación de las políticas neoliberales? RC24: Environmental justice, citizenship and governance, 1-4 de agosto, Buenos Aires, Argentina.

Musco, L., A. Cavallo, and A. Giangrande. 2004. I sillidi (Annelida, Polychaeta) dell litorale brindisino: possibilityá di un loro impiego come indicatori di qualitá dell ambiente. Thalassia Salentina 27:161-174.

Oksanen, J., F. G. Blanchet, R. Kindt, P. Legendre, P. R. Minchin, R. B. O'Hara, G. L. Simpson, P. Solymos, M. Henry, H. Stevens, and H. Wagne. 2013. vegan: Community Ecology Package. R package version 2.0-4. cran.r-project.org/ web/packages/vegan.

OSSE. 2014. www.mardelplata.gob.ar/Noticias/inauguraron-el-emisario.

Pielou, E. C. 1969. An introduction to mathematical ecology. Wiley-Interscience, New York. Pp. 286.

Pocklington, P., and P. G. Wells.1992. Polychaetes: key taxa for marine quality monitoring. Marine Pollution Bulletin 24:593-598.

Quiroga, S. Y., M. Bolaños, and M. K. Litvaitis. 2004. Policládidos (Platyhelminthes: “Turbellaria”) del Atlántico Tropical Occidental. Biota Colombiana 5(2):159-172.

Salas, H. J. 2000. Emisarios submarinos alternativa viable para la disposición de aguas negras de ciudades costeras en América latina y El Caribe. Centro Panamericano de ingeniería sanitaria y ciencias del ambiente. Río de Janeiro, Brasil. Journal WPCF 51:951-957.

Sánchez, M. A. 2014. Aplicación de un diseño BACI para la evaluación de impacto antrópico mediante poliquetos indicadores y la estructura de la comunidad intermareal de Brachidontes rodriguezii. Tesis doctoral. Universidad Nacional de Mar del Plata, Buenos Aires, Argentina. Pp. 206.

Sánchez, M. A., M. L. Jaubet, G. V. Garaffo, and R. Elías. 2013. Spatial and long-term analysis on reference and sewageimpacted sites of the SW Atlantic $\left(38^{\circ} \mathrm{S}, 57^{\circ} \mathrm{W}\right)$ to assess sensitive and tolerant polychaetes. Marine Pollution Bulletin 74:325-333.

Scagliola, M., P. Furchi, G. Von Haeften, A. P. Comino, E. Moschione, R. Gonzales, G. Gayoso, A. Caldararo, G. Cerda, S. Vergara, G. Genga, R. Elías, and E. A. Vallarino. 2006. Sewage outfall project of Mar del Plata city (Argentina): an effective intervention to achieve quality objectives on the marine environment. En: Annals of the 4th International Conference on Marine Waste Water Disposal and Marine Environment. Antalya (Turquía), 6-10 November. Pp. 22.

Shannon, C. E., and W. Weaver. 1963. The Mathematical Theory of Communication. Urbana, University of Illinois Press. Pp. 117.

Underwood, A. J. 1997. Experiments in ecology: Their Logical Design and Interpretation Using Analysis of Variance. Cambridge University press, Cambridge. Pp. 504.

Vallarino, E. A., M. S. Rivero, M. C. Gravina, and R. Elías. 2002. The community level response to sewage impact in intertidal mussel beds of the Southwestern Atlantic, and the use of the Shannon index to assess pollution. Revista de Biología Marina y Oceanografía 37:25-33.

Vallarino, E. A., and R. Elías. 2006. High-diverse lowly variable sewage-impacted community, low-diverse highly variable natural community: The paradox of the intertidal mussel beds of temperates areas of the SW Atlantic ( $38^{\circ}$ $\left.\mathrm{S}, 57^{\circ} \mathrm{W}\right)$. Current Trends in Ecology 1:77-91.

Vallarino, E. A. 2013. Contaminación intermareal por vertidos urbanos en Mar del Plata. Editorial académica Española. Pp. 196.

Zajac, R. N., and R. B. Whitlatch. 1982. Response of estuarine infauna to disturbance. II. Spatial and temporal variation of succession. Marine Ecology Progress Series 10:15-27.

Zar, J. H. 1999. Biostatistical Analysis, 4ta Edición. Prentice-Hall: Upper Saddle River, NJ. Pp. 662. 